\title{
Procedimentos Fisioterapêuticos para Disfunção Vésico-Esfincteriana de Pacientes com Traumatismo Raquimedular - Revisão Narrativa
}

\author{
Physiotherapeutic Procedures for Vesical - Sphincter Disorder in \\ Patients with Spinal Cord Trauma. A Narrative Bibliographic Review
}

Márcia Maria Gimenez ${ }^{1}$, Sissy Veloso Fontes², Marcia Maiumi Fukujima ${ }^{3}$

\begin{abstract}
RESUMO
A disfunção vésico-esfincteriana é comum no traumatismo raquimedular (TRM) e, pode ser dividida em bexiga neurogênica não-inibida, bexiga neurogênica reflexa, bexiga neurogênica autônoma, bexiga neurogênica paralítico-motora e bexiga neurogênica paralítico-sensitiva. Apresentamos uma revisão bibliográfica narrativa com análise crítica, sobre procedimentos fisioterapêuticos nas disfunções vésicoesfincterianas decorrente do TRM. Foram encontrados ao todo 14 artigos, sendo estes estudos analíticos. Os procedimentos fisioterapêuticos discutidos subdividem-se de acordo com o tipo de bexiga. Atualmente, os procedimentos mais utilizados são as manobras e técnicas de esvaziamento vesical, terapia comportamental com ênfase no esvaziamento vesical cronometrado, exercícios de contração isométrica e isotônica das musculaturas adjacentes à bexiga, exercícios de Kegel (exercícios para o assoalho pélvico) e, Biofeedback (utiliza-se tanto o eletromiográfico como o de pressão) e a acupuntura, eletroacupuntura e eletroestimulação. Contudo, de todos trabalhos levantados observou-se que nenhum deles possui nível de evidência suficiente para comprovar os reais efeitos do tratamento fisioterapêutico para disfunções vésico-esfincteriana decorrente do TRM, sendo necessários novos estudos com desenhos metodológicos mais adequados.
\end{abstract}

Unitermos: Bexiga Neurogênica, Traumatismo raquimedular, Fisioterapia, Incontinência urinária, Disfunção vésico-esfincteriana.

Citação: Gimenez MM, Fontes SV, Fukujima MM. Procedimentos fisioterapêuticos para disfunção vésico-esfincteriana de pacientes com traumatismo raquimedular - revisão narrativa. Rev Neurociencias 2005; 13(1):034-038.

\section{SUMMARY}

The vesical - sphincter disorder is usual in the spinal cord trauma (SCT) and may be divided in non inhibited neurogenic bladder, reflex neurogenic bladder, autonomic neurogenic bladder, motor - paralytic neurogenic bladder and paralytic - sensitive neurogenic bladder. This study has as research method, a narrative bibliographic review with critical analysis of scientific studies about physiotherapeutic procedures for vesical - sphincter disorder due SCT. There was founded 14 scientific studies in the searched literature, where was analytical. Of the 14 analytical studies, one was systematic reviews, two randomised clinical trials, four case control studies and eight bibliographic reviews. The physiotherapeutic procedures discussed

Trabalho realizado: Universidade Federal de São Paulo - Escola Paulista de Medicina

1 - Fisioterapeuta, Pós-Graduanda da UNIFESP

2 - Fisioterapeuta, Educadora Física, Mestre em Neurociências e Doutora em Ciências pela UNIFESP

3 - Neurologista, Mestre em Neurologia pela UNIFESP

Endereço para correspondência: Dra. Márcia Maiumi Fukujima

Universidade Federal de São Paulo , Departamento de Neurologia/Neurocirurgia, Disciplina de Neurologia Clínica - UNIFESP

Rua Botucatu 740 -Vila Clementino - São Paulo

04023-900, SP - Brasil - Telefone: 115575.5240

E-mail: maiumi@neuro.epm.br 
are subdivided in accordance with the bladder type. Nowadays, the most used procedures are maneuvers and techniques of vesical emptying, incited and measured vesical emptying, isometric and isotonic contraction exercises of bladder adjacent muscles, Kegel exercises (for pelvic floor), Biofeedback (either electromiographic or of pressure), acupuncture and stimulation electrical. However, of all searched works, we observed that none of them have evidence levels enough to show the effects of physiotherapeutic treatment for vesical - sphincter disorders due SCT. Rev. Neurociências 2005; 13(1):000-000.

\section{Keywords: Neurogenic bladder, Spinal cord injury, Physical therapy, Urinary incontinence, Vesical-sphincter disorder.}

Citation: Gimenez MM, Fontes SV, Fukujima MM. Physiotherapeutic procedures for vesical - sphincter disorder in patients with spinal cord trauma - a narrative bibliographic review. Rev Neurociencias 2005; 13(1):034-038.

O traumatismo raquimedular é uma das mais graves síndromes incapacitantes, sendo as disfunções urológicas advindas deste trauma uma das significantes causas de mortalidade destes pacientes, mesmo com os avanços atuais na área da saúde para intervenção nestas disfunções ${ }^{1}$.

O controle vesical é realizado pelo sistema nervoso coordenando os mecanismos voluntários e involuntários da micção. A disfunção vesical resulta na bexiga neurogênica. Várias doenças neurológicas causam disfunção vésico-esfincteriana, entretanto a causa mais comum de bexiga neurogênica no adulto é o traumatismo raquimedular ${ }^{2}$

O tipo de disfunção varia de acordo com o nível e extensão da lesão medular. O controle medular da micção é na região de S2 - S4 da medula espinhal, alojado nos corpos vertebrais T12 - L1. As lesões acima deste nível são caracterizadas por bexigas neurogênicas nãoflácidas (bexiga neurogênica reflexa e bexiga neurogênica não-inibida) e as lesões no centro da micção ou abaixo dele são denominadas bexigas neurogênicas flácidas (bexiga neurogênica autônoma, bexiga neurogênica paralítico-sensitiva e bexiga neurogênica paralítico-motora) ${ }^{3}$.

O tratamento de qualquer tipo de bexiga neurogênica é guiado pela necessidade de promover o esvaziamento vesical completo, preservar o bom funcionamento renal, evitar complicações e, principalmente permitir a inclusão social e profissional do paciente ao adquirir a reeducação urinária².

Na última década, houve avanços na área da saúde que trabalha em prol das pessoas portadoras de deficiência física, dentre eles o lesado medular. A fisioterapia, ciência da saúde que estuda, previne e trata os distúrbios cinéticos funcionais, intercorrentes em órgãos e sistemas do corpo humano, tem buscado em estudos, metodologicamente mais adequados comprovar seus reais efeitos, indicações e benefícios. Porém, pouco sabe-se a respeito de técnicas fisioterapêuticas para tratamento de bexiga neurogênica em lesado medular.

O objetivo deste trabalho foi discutir os estudos sobre o tratamento fisioterapêutico para as disfunções vésicoesfincteriana decorrente do traumatismo raquimedular.

Foram encontrados ao todo 14 artigos (Quadro 1), todos analíticos (uma revisão sistemática, dois ensaios clínicos randomizados, quatro estudos tipo casos e controles e oito revisões descritivas).

\section{Bexiga Neurogênica Não-Inibida}

Neste tipo de bexiga, o enchimento vesical desencadeia contrações não inibidas, que passam a ser interpretadas como urgência miccional ou incontinência urinária, tornando a capacidade vesical reduzida ${ }^{4}$.

Os exercícios de Kegel incluem-se no tratamento, consistem na contração voluntária da musculatura do assoalho pélvico, com o objetivo de impedir a incontinência urinária. Neste tipo de bexiga o objetivo principal é a inibição do desejo de urinar, que só é possível com a inervação integra do músculo do assoalho pélvico. O paciente deve ser orientado a realizar os exercícios que ajudam na força muscular, na resistência uretral e na pressão de fechamento uretral varias vezes ao dia ${ }^{5}$.

O Biofeedback com eletromiografia também é um recurso bastante utilizado pela fisioterapia em pacientes portadores de incontinência urinária, pois os músculos do períneo ou assoalho pélvico formam um apoio elástico para o conteúdo pélvico e abdominal. Esta técnica treina os pacientes quanto ao desenvolvimento de maior sensação de controle e domínio vesical ${ }^{5}$.

Pode-se utilizar tanto o biofeedback eletromiográfico como o de pressão. O primeiro possui um sensor que detecta a atividade elétrica liberada pelos músculos quando contraem-se, esse sinal é amplificado e processado de forma visual ou sonora para o paciente. O biofeedback de pressão utiliza uma sonda inflável colocado no interior da vagina ou do ânus (mulheres virgens ou homens) e registra a variação de pressão que é mostrada ao paciente, sendo útil para melhorar a visão cinestésica da região6.

Na revisão sistemática de treinamento vesical para incontinência urinária em adultos, concluiu-se que pode 


\begin{tabular}{|c|c|c|c|}
\hline AUTORES & TIPO/ESTUDO & № pts & COMENTÁRIOS \\
\hline Eustice et al, 2002 & revisão sistemática & 355 & $\begin{array}{l}\text { verificaram a importância dos efeitos do } \\
\text { esvaziamento vesical cronometrado e incitado }\end{array}$ \\
\hline Cheng et al, 1998 & ensaio clínico randomizado & 80 & $\begin{array}{l}\text { avaliar a acupuntura em bexiga neurogênica por } \\
\text { lesão da medula espinal - relatório preliminar } \\
\text { relatando os benefícios com a técnica. }\end{array}$ \\
\hline Yamanishi et al, 2000 & ensaio clínico randomizado & 68 & $\begin{array}{l}\text { ensaio clínico randomizado duplo-cego de } \\
\text { eletroestimulação para incontinência urinária por } \\
\text { hiperreflexia do detrusor }\end{array}$ \\
\hline Gomes, 1998 & estudo de casos e controles & 20 & $\begin{array}{l}\text { realizar trabalho muscular isométrico no tratamento } \\
\text { da reeducação vesical em portadores de bexiga } \\
\text { neurogênica flácida decorrente ao choque medular } \\
\text { pós-traumatico verificando } 70 \% \text { de melhora dos pacientes }\end{array}$ \\
\hline Hongo et al, 2000 & estudo de casos e controles & 13 & $\begin{array}{l}\text { acupuntura para os sintomas clínicos e } \\
\text { mensurações hemodinâmicas em pacientes com } \\
\text { lesão da medula espinal com hiperreflexia do detrusor. }\end{array}$ \\
\hline Weiss, 2001 & estudo de casos e controles & 52 & $\begin{array}{l}\text { técnica miofacial em pacientes com urgência e aumento da } \\
\text { freqüência urinária característica da bexiga hiperativa }\end{array}$ \\
\hline Amarenco et al, 2003 & estudo de casos e controles & 44 & $\begin{array}{l}\text { comparando as cistometrias antes e após a eentp. } 21 \\
\text { pacientes ocorreram aumento em } 50 \% \text { ou mais no } \\
\text { volume no momento da contração não inibida, } 13 \\
\text { aumentaram mais de } 100 \mathrm{ml} \text { o volume da } 1^{\text {a }} \text { contração, } 15 \\
\text { melhoraram mais de } 50 \% \text { da ccm e } 1 \text { melhorou a ccm sem } \\
\text { aumentar o volume na contração não inibida. }\end{array}$ \\
\hline Fakacs et al, 1992 & revisão de literatura & & reabilitação das bexigas neurogênicas \\
\hline Bo et al, 2000 & revisão de literatura & & $\begin{array}{l}\text { tratamento não farmacológico para bexiga hiperativa- } \\
\text { exercícios do assoalho pélvico }\end{array}$ \\
\hline Payne, 2000 & revisão de literatura & & terapia comportamental para bexiga hiperativa \\
\hline Vapnek, 2001 & revisão de literatura & & tratamento das disfunções urinárias \\
\hline Roe et al, 2002 & revisão de literatura & & $\begin{array}{l}\text { treino vesical em pacientes adultos com incontinência } \\
\text { urinária }\end{array}$ \\
\hline Sakakibara et al, 2001 & revisão de literatura & & $\begin{array}{l}\text { crise de hiperreflexia autonômica em pacientes com } \\
\text { bexiga neurogênica }\end{array}$ \\
\hline Magaldi et al, 2002 & revisão de literatura & & $\begin{array}{l}\text { fisioterapia uroginecológica: incontinência urinária e } \\
\text { prolapso }\end{array}$ \\
\hline
\end{tabular}

Quadro 1. Literatura sobre tratamento fisioterapêutico da bexiga neurogênica após TRM

ser útil no tratamento da incontinência urinária, mas não há evidências suficientes para mostra se a terapia com fármacos é melhor que o treino vesical ${ }^{7}$.

A fisioterapia miofascial é a técnica que utiliza alongamento e compressão dos pontos de gatilhos "trigger points" no assoalho pélvico, usado tanto para cistite intersticial, como para a incontinência urinária, comum na bexiga neurogênicå .

A terapia comportamental com o esvaziamento vesical incitado a cada 2 horas é eficaz a curto prazo, sendo melhor do que quando não realiza-se nenhuma terapia e, o esvaziamento incitado associado com outra terapia é mais efetivo do que quando realizado somente o esvaziamento vesical incitado para a incontinência urinária, porém ainda há necessidade de mais pesquisas sobre o assunto 9 .

No esvaziamento vesical cronometrado, o incontinente pode ser educado sobre a condição de desenvolver estratégias para minimizar ou eliminar a incontinência. O programa consiste na observação para cronometração de episódios de incontinência por 2 a 3 dias, de modo a avaliar o padrão de esvaziamento. Um esquema de esvaziamento é, então estabelecido em intervalos periódicos. Esta terapia pode ou não estar associada ao uso de fármacos. O método de esvaziamento vesical cronometrado é, demonstravelmente efetivo, com taxas de melhoria em episódios de incontinência por hiperreflexia do músculo detrusor ${ }^{10}$.

Amarenco et al, 2003 realizaram um estudo com uso da eletroestimulação do nervo tibial posterior associado ao uso concomitante do estudo urodinâmico, para este estudo foram inclusos 44 pacientes destes 37 tinham bexiga hiperativa neurogênica sendo $15 \mathrm{com}$ lesão da medula espinal, 13 esclerose múltipla e 9 Parkinson. O objetivo foi verificar a mudança dos resultados urodinâmicos, quanto ao volume vesical onde ocorria a primeira contração nãoinibida do músculo detrusor e capacidade cistométrica máxima, no mesmo instante, o qual o paciente estava realizando a eletroestimulação do nervo tibial posterior e concluíram que a eletroestimulação do nervo tibial posterior sugere uma melhora objetiva dos resultados urodinâmicos, tornando essa proposta encorajadora para o tratamento não invasivo na prática clínica ${ }^{11}$. 


\section{Bexiga Neurogênica Reflexa}

Este tipo de bexiga é causada por lesão total acima do centro de micção, com comprometimento sensitivo e motor, tendo como característica a dissinergia detrusor esfincteriana ${ }^{4}$.

Para este tipo de bexiga neurogênica foram encontradas as seguintes técnicas: A técnica de percussão, o amassamento, o tracionamento dos pêlos púbicos para estimular reflexamente $\mathrm{o}$ ato da micção ${ }^{12}$.

A acupuntura é uma modalidade da tradicional medicina chinesa e tem sido utilizada para os sintomas clínicos e mensurações hemodinâmicas em pacientes com lesão da medula espinhal que apresentam hiperreflexia do detrusor. As agulhas de acupuntura foram inseridas bilateralmente nos pontos B-33 (Zhongliao), padronizados pela Organização Mundial da Saúde, sobre a pele do terço posterior do forame sacral em direção cranial. A agulha foi inserida em cada lado do forame em profundidade suficiente para que, sua ponta fosse colocada próxima ao periósteo sacral (50-60mm), e, então as agulhas bilaterais eram rodadas recíproca e manualmente num mesmo eixo, por 10 minutos. O tratamento foi repetido uma vez por semana durante 4 semanas. Este estudo foi o primeiro a relatar os resultados da acupuntura em pacientes com lesão da medula espinhal com incontinência urinária causada pela hiperreflexia do detrusor. Na maioria dos pacientes, a acupuntura bilateral sobre os pontos B-33 melhorou, significantemente a capacidade da bexiga, finalmente, deve-se notar que o número de pacientes presente no estudo é pequeno e que, as estatísticas sob estas condições podem ser questionadas ${ }^{13}$. Esta técnica também pode ser utilizada em outros tipos de bexiga neurogênica, pois, quando gira-se a agulha para direita tonifica-se e quando girase a agulha para esquerda inibe-se o músculo detrusor.

Foi realizado um ensaio clínico de acupuntura para bexiga neurogênica por lesão da medula espinhal utilizando o método de eletroacupuntura. Os autores relataram que em cada ponto da acupuntura existe altas concentrações de neurotransmissores e hormônios nos seres humanos e estes hormônios podem causar inibição, excitação ou ambos para os neurônios designados. A eletroacupuntura pode ativar fibras do nervo aferente, podendo iniciar vários mecanismos fisiológicos. Neste estudo foi observado que os pacientes com lesão medular completa não conseguem que a bexiga alcance o mecanismo funcional adequado. Este estudo teve como método a seleção de quatro pontos de acupuntura, para administração da bexiga neurogênica em pacientes com traumatismo raquimedular. Este estudo concluiu que a função vesical com a eletroacupuntura melhorou, porém o grupo controle chegou a alcançar esta melhora só que demorou mais, sendo assim, estudos adicionais devem ser realizados. Esta técnica também pode ser realizada em outros tipos de bexiga neurogênica podendo utilizar pontos de inibição ou excitação do músculo detrusor ${ }^{14}$.
Em um ensaio clínico randomizado sobre a eletroestimulação na incontinência urinária decorrente da hiperreflexia do detrusor, verificou-se que esta técnica possui, como mecanismo à excitação elétrica para inibição do detrusor, a inibição reflexa eferente do nervo pélvico ou ativação eferente do hipogástrio por excitação aferente do nervo pudendo. A excitação ativa os interneurônios espinhais que lançam neurotransmissores inibitórios. Foi concluído que, a excitação elétrica é um tratamento efetivo para incontinência urinária, devido a hiperreflexia do detrusor, pois, esta hiperreflexia de detrusor desapareceu em $25 \%$ dos pacientes e melhorou em $62.5 \%$ demonstrando a efetividade da terapia ${ }^{15}$

Um levantamento bibliográfico sobre a atuação da fisioterapia nas diversas causas de incontinência urinária, inclusive por hiperreflexia do detrusor e, as técnicas mais utilizadas são a estimulação elétrica, treinamento da musculatura do assoalho pélvico com ou sem o uso do biofeedback. Muitas vezes, estes tratamentos estão associados ao uso de fármacos, os autores concluíram que o uso destas terapias é eficaz a curto prazo, salientando a necessidade de uma pesquisa referente à sua efetividade a longo prazo e, também o estudo das técnicas individualmente ${ }^{16}$.

Para este tipo de bexiga também podem ser utilizadas como coadjuvante as terapias comportamentais.

É de extrema importância à atenção que o fisioterapeuta deve ter ao realizar manobras e técnicas de reeducação vesical em pacientes com lesão medular acima de T6, pois eles podem desencadear uma hiperreflexia autonômica, caso o paciente desencadeie esta crise devese colocá-lo sentado para diminuir a pressão arterial, o esvaziamento vesical deve ser realizado com cateter, pesquisar a presença de fezes na região anoretal, remover o estímulo tátil, doloroso ou térmico, estas são medidas não-farmacológicas. Se o quadro não reverter, deve-se então utilizar medidas farmacológicas. O paciente pode necessitar da intervenção clínica ministrada por uma equipe interdisciplinar ${ }^{17}$.

\section{Bexiga Neurogênica Autônoma}

É o tipo principal tipo de bexiga neurogênica flácida, sendo causada por lesão abaixo ou no centro da micção interrompendo o arco-reflexo. Este tipo de bexiga é comum na fase de choque medular ${ }^{4}$. Neste caso utilizam-se as seguintes manobras: a manobra de valsalva, a manobra de credé e o ato de tossir.

Segundo Gomes (1998), uma outra modalidade de tratamento para este tipo de bexiga é o uso de contrações isométricas dos músculos que encontram-se adjacentes à bexiga, alguns favorecerão a profilaxia circulatória e, outros proporcionarão melhora da função respiratória. A estimulação da bexiga ocorre pela pressão exercida no trabalho isométrico. A indicação terapêutica pode ser de- 
vido à contração muscular isométrica (na instabilidade da fratura) e a contração muscular isotônica (na estabilidade) aplicando, assim a cinesioterapia. O autor salienta uma ordem na seqüência do tratamento, como segue: inicia-se com a dessensibilização, para proporcionar a acomodação de receptores, aumentando o limiar de excitabilidade, dando alívio ao paciente, podendo, assim iniciar a cinesioterapia $^{18}$.

A técnica consiste em orientar o paciente a contrair a parede abdominal e manter por alguns segundos. $\mathrm{O}$ músculo íliopsoas em sua contração isométrica provoca a compressão das paredes laterais da musculatura da bexiga, gerando um estímulo desta parede. A contração isométrica da musculatura Glútea proporciona, posteriormente à estabilidade do quadril na deambulação e uma retroversão da pelve, aumentando a pressão intravisceral, conseqüentemente melhorando a estimulação da bexiga. A contração isométrica do músculo Grande Dorsal proporciona a estabilização da coluna tóraco-Iombar-sacral e auxilia o trabalho da musculatura abdominal. Com a aplicação deste tratamento $70 \%$ dos pacientes obtiveram a retomada da funcionalidade da bexiga ${ }^{18}$.

Neste tipo de bexiga também podem ser realizadas as seguintes técnicas já descritas anteriormente como o biofeedback, acupuntura, eletroacupuntura, terapia comportamental.

\section{Bexiga Neurogênica Paralítico-Sensitiva e Bexiga Neurogênica Paralítica Motora}

A bexiga neurogênica paralítico sensitiva consiste em um subtipo de bexiga neurogênica flácida, porém a lesão é limitada ao corno posterior da medula espinhal. E a bexiga neurogênica paralítico-motora é caracterizada por lesão dos neurônios ou nervos que controlam a bexiga ${ }^{4}$.

Assim como na bexiga neurogênica autônoma, a reeducação vesical da bexiga neurogênica paralítica sensitiva e bexiga neurogênica motora, necessitam de manobras que aumentem a pressão intravesical para desencadear a micção, utilizando-se a manobra de valsalva, crede, estímulo de tosse, contração muscular isométrica e isotônica da musculatura abdominal, do músculo íliopsoas, da musculatura glútea e musculatura paravertebral, acupuntura, eletroacupuntura, terapia comportamental com o esvaziamento vesical incitado e cronometrado, exercícios de kegel e biofeedback.

Concluindo, os procedimentos fisioterapêuticos discutidos subdividem-se de acordo com o tipo de bexiga. Atualmente, os procedimentos mais utilizados são as manobras e técnicas de esvaziamento vesical, terapia comportamental com o esvaziamento vesical incitado e cronometrado, exercícios de contração isométrica e isotônica das musculaturas adjacentes à bexiga, exercícios de Kegel (exercícios para o assoalho pélvico) e, Biofeedback (utilizando-se tanto com eletromiográfico como o de pressão) e a acupuntura, eletroacupuntura e eletroestimulação.

Porém, observou-se que os níveis de evidência são insuficientes para demonstrar os efeitos dos procedimentos fisioterapêuticos nas diversas disfunções vésicoesfincteriana. Sendo assim, novos ensaios clínicos devem ser realizados.

\section{REFERÊNCIAS BIBLIOGRÁFICAS}

1. D'ancona CAL. Distúrbios Neurológicos da Bexiga e Urodinâmica. In: Hering FLO, Srougi M. Urologia - Diagnóstico e Tratamento. São Paulo: Roca; 1998

2. Yamauti AY. Cuidados de Enfermagem em Pacientes Portadores de Lesão Medular. In: Greve JMD, Casalis MEP, Barros Filho TEP. Diagnóstico e Tratamento da Lesão da Medula Espinhal. São Paulo: Roca; 2001.

3. Lapides J. Urologia. Rio de Janeiro: Interamericana; 1992.

4. Gandolpho L, Heilberg, IP, Cuppoloni MM. Atualização em Infecções Urinárias: Bexiga Neurogênica. São Paulo: Moreira Jr.; 1995.

5. Vapnek JM. Urinary Incontinence: Screening and Treatment of Urinary Dysfunction. Geriatrics 2001;56,10:25-32.

6. Magaldi C, Araújo RC, Pacetta AM. Fisioterapia Uroginecológica: Incontinência Urinária e Prolapso. O COFFITO 2002; 28-32.

7. Roe B, Williams K, Palmer M. Bladder training for urinary incontinence in adults. Cochrane Database Syst Rev 2004; (1):CD001308.

8. Weiss JM. Pelvic Floor Myofascial Trigger Points: Manual Therapy for Interstitial Cystitis and The Urgency-Frequency Syndrome. J Urol 2001;166:2226-2231.

9. Eustice S, Roe B, Paterson J. Prompted voiding for the management of urinary incontinence in adults. Cochrane Database Syst Rev 2000(2):CD002113.

10. Payne CK. Behavioral Therapy for Overactive Bladder. Urology 2000;55(Suppl 5A): 3-6.

11. Amarenco G, Sheik Ismael A, Even-Schneider O, et al. Urodynamic effect of acute transcutaneous posterior tibial nerve stimulation in overactive bladder. J Urol 2003;169:2210-2215.

12. Fakacs $C$, Audic B, Bournaud M, et al. Rehabilitación de Las Vejigas Neurógenas. Kinésithérapie 1992;26:1-29.

13. Hongo $H$, Naya $Y$, Ukimura $O$, et al. Acupunture on Clinical Symptoms and Urodynamic Measurements in Spinal Cord Injured Patients with Detrusor Hyperreflexia. Urol Int 2000;65:190-195

14. Cheng PT, Wong MK, Chang PL. A Therapeutic Trial of Acupunture in Neurogenic Bladder of Spinal Cord Injured Patients - a preliminary report. Spinal Cord 1998;36:476-480.

15. Yamanishi T, Yasuda K, Sakakibara R et al. Randomized, DoubléBlind Study of Electrical Stimulation for Urinary Incontinence Due to Detrusor Overactivity. Urology 2000;55:353-357.

16. Bo K, Berghmans LCM. Nonpharmacologic Treatments for Overactive Bladder - Pelvic Floor Exercises. Urology 2000;55(suppl. 5A):7-11

17. Sakakibara R, Uchiyama T, Kuwabara $S$, et al. Autonomic Dysreflexia Due to Neurogenic Bladder Dysfunction; a Unusual Presentation of Spinal cord Sarcoidosis. J Neurol 2001;71(6):819-823.

18. Gomes LG. O Trabalho Muscular Isométrico no Tratamento da Reeducação Vesical em Portadores de Bexiga Neurogênica Flácida Decorrente ao Choque Medular Pós-Traumático. Fisioterapia em Movimento 1998;11(1):49-90. 